\title{
SOME ASYMPTOTIC RESULTS FOR THE PERIODOGRAM OF A STATIONARY TIME SERIES
}

\author{
A. M. WALKER
}

(received 27 August 1964)

\section{Introduction}

Let $x_{1}, x_{2}, \cdots, x_{n}$ be $n$ consecutive observations generated by a stationary time series $\left\{x_{t}\right\}, t=0, \pm 1, \pm 2, \cdots$, with $E\left(x_{t}^{2}\right)<\infty$. The periodogram of the set of observations, which may be defined as a function $I_{n}$ of angular frequency with range $[0, \pi]$ that is proportional to $\left|\sum_{t=1}^{n} x_{t} e^{i \omega t}\right|^{2}$, plays an important part in methods of making inferences about the structure of $\left\{x_{i}\right\}$, particularly its spectral distribution function or spectral density. The distributional properties of $I_{n}$ consequently have been studied fairly thoroughly in recent years; an account of the principal results can be found, for example, in Bartlett (1955 Chapter 9), and Hannan (1960 Chapters 3, 4). In this paper we show that certain important asymptotic theorems can be proved quite simply with complete rigour under suitable conditions; it is believed that most of the proofs are substantially simpler than those that have previously been given. We also consider to what extent the conditions can be weakened and still admit tolerably simple proofs. Finally we discuss an interesting question which is so far largely unsolved, namely the determination of properties of the distributions of $\max _{0 \leqq \omega \leqq \pi} I_{n}(\omega)$ and $\max _{0 \leqq \omega \leqq \pi}\left\{I_{n}(\omega) / 2 \pi f(\omega)\right\}$, where $f(\omega)$ denotes the spectral density of $\left\{x_{t}\right\}$.

Following Bartlett (1955) and Hannan (1960) we shall use the definition

$$
I_{n}(\omega)=(2 / n)\left|\sum_{t=1}^{n} x_{t} e^{i \omega t}\right|^{2} .
$$

We shall also assume that $\left\{x_{t}\right\}$ is a linear process, such that

$$
x_{t}=\sum_{u=0}^{\infty} \alpha_{u} \varepsilon_{t-u}
$$

where the $\varepsilon_{t}$ are independently and identically distributed with $E\left(\varepsilon_{t}\right)=0$, $E\left(\varepsilon_{t}^{2}\right)=1$, and the $\alpha_{u}$ are constants satisfying the condition

$$
\sum_{u=0}^{\infty}\left|\alpha_{u}\right|<\infty
$$


In the usual definition of a linear process (Bartlett, 1955, p. 146; Hannan, 1960, p. 33) it is assumed that $\sum_{u=0}^{\infty} \alpha_{u}^{2}<\infty$. However, (3) is quite a weak restriction, and incidentally ensures that the spectral density $f(\omega)$ of $\left\{x_{i}\right\}$ exists and is continuous for all $\omega$, being given by

$$
2 \pi f(\omega)=\left|\sum_{u=0}^{\infty} \alpha_{u} e^{i \omega u}\right|^{2}
$$

\section{The asymptotic relation}

between the periodogram of a linear process

and the periodogram of the corresponding residual process

We first consider the relation between

$$
I_{n, x}(\omega) \text { and } I_{n, \varepsilon}(\omega)=(2 / n)\left|\sum_{t=1}^{n} \varepsilon_{t} e^{i \omega t}\right|^{2},
$$

the corresponding value of the periodogram of the 'residual' process $\left\{\varepsilon_{t}\right\}$.

THEOREM 1. Let

$$
\begin{aligned}
& J_{n, x}(\omega)=(2 / n)^{\frac{1}{2}} \sum_{t=1}^{n} x_{t} e^{i \omega t}, \\
& J_{n, \varepsilon}(\omega)=(2 / n)^{\frac{1}{2}} \sum_{t=1}^{n} \varepsilon_{t} e^{i \omega t}, \\
& R_{n}(\omega)=J_{n, x}(\omega)-A(\omega) J_{n, \varepsilon}(\omega)
\end{aligned}
$$

where

and

$$
A(\omega)=\sum_{u=0}^{\infty} \alpha_{u} e^{i \omega u}
$$

$$
T_{n}(\omega)=I_{n, x}(\omega)-|A(\omega)|^{2} I_{n, \varepsilon}(\omega)
$$

Then for each $\omega$,

$$
p \lim _{n \rightarrow \infty} R_{n}(\omega)=0
$$

and

$$
p \lim _{n \rightarrow \infty} T_{n}(\omega)=0 .
$$

Proof. Substituting (2) in (5) and changing the order of summation with respect to $t$ and $u$, we obtain

$$
J_{n, x}(\omega)=\sum_{u=0}^{\infty} \alpha_{u} e^{i \omega u} \sum_{v=1-u}^{n-u} \varepsilon_{v} e^{i \omega v}
$$


Hence if

$$
\begin{aligned}
& Z_{n, u}(\omega)=\left\{\sum_{1-u}^{n-u}-\sum_{1}^{n}\right\} \varepsilon_{v} e^{i \omega v}, \\
& R_{n}(\omega)=(2 / n)^{\frac{1}{2}} \sum_{u=1}^{\infty} \alpha_{u} e^{i \omega u} Z_{n, u}(\omega) .
\end{aligned}
$$

Suppose that $0<\omega<\pi$. Then from the definition (11) we have

$$
\left.\begin{array}{rl}
E\left\{\left|Z_{n, u}(\omega)\right|^{2}\right\} & =2 u, \quad 1 \leqq u<n \\
& =2 n, \quad u \geqq n
\end{array}\right\} .
$$

Therefore

$$
\begin{aligned}
E\left\{\left|R_{n}(\omega)\right|\right\} & \leqq(2 / n)^{\frac{1}{2}} \sum_{u=1}^{\infty}\left|\alpha_{u}\right| E\left|Z_{n, u}(\omega)\right| \\
& \leqq(2 / n)^{\frac{1}{2}} \sum_{u=1}^{\infty}\left|\alpha_{u}\right|\left[E\left\{\left|Z_{n, u}(\omega)\right|^{2}\right\}\right]^{\frac{1}{2}} \\
& =2\left\{\sum_{u=1}^{n-1}\left|\alpha_{u}\right|(u \mid n)^{\frac{1}{2}}+\sum_{u=n}^{\infty}\left|\alpha_{u}\right|\right\} .
\end{aligned}
$$

Now when $n \rightarrow \infty$ the second sum in (14) obviously tends to zero, and so also does the first sum, since it is not greater than

$$
\sum_{u=k(n)+1}^{n-1}\left|\alpha_{u}\right|+\{k(n) / n\}^{\frac{1}{2}} \sum_{u=1}^{k(n)}\left|\alpha_{u}\right|
$$

and $k(n)$ can be chosen so that $\lim _{n \rightarrow \infty}\{k(n) / n\}=0, \lim _{n \rightarrow \infty} k(n)=\infty$. Hence

$$
\lim _{n \rightarrow \infty} E\left\{\left|R_{n}(\omega)\right|\right\}=0,
$$

which is a sufficient condition for (9).

If $\omega=0$ or $\pi$ the same argument applies except that the factor 2 does not appear in the expression for $E\left\{\left|Z_{n, u}(\omega)\right|\right\}^{2}$.

(10) then follows immediately since

$$
\begin{aligned}
T_{n}(\omega) & =\left|R_{n}(\omega)+A(\omega) J_{n, \varepsilon}(\omega)\right|^{2}-|A(\omega)|^{2} I_{n, \varepsilon}(\omega) \\
& =R_{n}(\omega) A^{*}(\omega) J_{n, \varepsilon}^{*}(\omega)+R_{n}^{*}(\omega) A(\omega) J_{n, \varepsilon}(\omega)+\left|R_{n}(\omega)\right|^{2},
\end{aligned}
$$

and

$$
\begin{aligned}
E\left\{\left|J_{n, \varepsilon}(\omega)\right|^{2}\right\} & =2 \text { if } 0<\omega<\pi \\
& =1 \text { if } \omega=0 \text { or } \pi,
\end{aligned}
$$

so that $J_{n, \varepsilon}(\omega)$ is $O_{p}(1)$.

Previous derivations of (9) and (10) have assumed that the $\alpha_{u}$ obey some stronger condition which enables a statement to be made about 
the order of magnitude of $R_{n}(\omega)$ or $T_{n}(\omega)$. For example, Hannan (1960, p. 54) uses the condition

$$
\sum_{u=1}^{\infty} u \frac{1}{2}\left|\alpha_{u}\right|<\infty,
$$

under which (14) clearly gives

$$
E\left\{\left|R_{n}(\omega)\right|\right\}=O\left(n^{-\frac{1}{2}}\right),
$$

so that $R_{n}(\omega)$, and therefore (from (16)) $T_{n}(\omega)$, are both $O_{p}\left(n-\frac{1}{2}\right)$. (Hannan in fact obtains the slightly stronger results $E\left\{\left|R_{n}(\omega)\right|^{2}\right\}=O\left(n^{-1}\right)$, $E\left\{\left|T_{n}(\omega)\right|^{2}\right\}=O\left(n^{-1}\right)$; the former follows immediately by applying the Cauchy-Schwartz inequality to show that (13) is an upper bound to $\left[E\left\{\left|R_{n}(\omega)\right|\right\}^{2}\right]^{\frac{1}{2}}$, but the proof of the latter, which also requires the assumption that $E\left(\varepsilon_{t}^{4}\right)<\infty$, is less straightforward). However, in the main application of the relation between $J_{n, x}(\omega)$ and $J_{n, \varepsilon}(\omega)$, or between $I_{n, x}(\omega)$ and $I_{n, \varepsilon}(\omega)$, namely the determination of the asymptotic distribution of a finite set of periodogram values, these order of magnitude statements can be dispensed with (as we shall see in $\S 3$ ).

We now examine the behaviour of the largest difference between $I_{n, x}(\omega)$ and $|A(\omega)|^{2} I_{n, \varepsilon}(\omega)$ when $\omega$ runs through the whole set of angular frequency values. In practice, periodogram values are usually computed only for angular frequencies which are multiples of $2 \pi / n$, and we therefore consider first the case where $\omega$ is restricted to the discrete set $\left\{\omega_{j}\right\}$, $j=0,1, \cdots,[n / 2]$, with $\omega_{j}=2 \pi j / n$. To begin with, we shall also suppose that $\varepsilon_{t}$ has a normal distribution, so that $\left\{x_{t}\right\}$ becomes a Gaussian process. This assumption greatly simplifies the problem by enabling the distribution of the largest of the $I_{n, \varepsilon}\left(\omega_{j}\right)$ for $0<\omega_{j}<\pi$ to be determined exactly.

THEOREM 2. Let the distribution of $\varepsilon_{t}$ be normal, and let

$$
\sum_{u=1}^{\infty} u^{\frac{1+\delta}{2+\delta}}\left|\alpha_{u}\right|<\infty \text {, for some } \delta>0 .
$$

Then, if we write $m=[(n-1) / 2]$,

$$
p \lim _{n \rightarrow \infty} \max _{1 \leqq j \leqq m}\left|T_{n}\left(\omega_{j}\right)\right|=0 .
$$

COROLLARY.

$$
p \lim _{n \rightarrow \infty} \max _{0 \leqq j \leqq[n / 2]}\left|T_{n}\left(\omega_{j}\right)\right|=0 .
$$

Proof. From (16) we have

$$
\begin{aligned}
\max _{j}\left|T_{n}\left(\omega_{j}\right)\right| \leqq & 2 \max _{j}\left|R_{n}\left(\omega_{j}\right)\right| \max _{j}\left|A\left(\omega_{j}\right)\right| \max _{j}\left|J_{n, \varepsilon}\left(\omega_{j}\right)\right| \\
& \left.+\max _{j}\left|R_{n}\left(\omega_{j}\right)\right|\right\}^{2} .
\end{aligned}
$$


Now it is easily seen that the random variables $\frac{1}{2} I_{n, \varepsilon}\left(\omega_{g}\right), 1 \leqq j \leqq m$, are distributed independently and each have probability density $e^{-x}$ (see, for example, Hannan, 1960 , p. 76). Hence $\frac{1}{2} \max _{1 \leqq j \leqq m} I_{n, \varepsilon}\left(\omega_{j}\right)$ has distribution function $\left(1-e^{-x}\right)^{m}$, and so

$$
\begin{aligned}
\frac{1}{2} E\left\{\max _{1 \leq j \leq m}\right. & \left.I_{n, \varepsilon}\left(\omega_{j}\right)\right\}=\int_{0}^{\infty}\left\{1-\left(1-e^{-x}\right)^{m}\right\} d x \\
& =\int_{0}^{1}\left(\frac{1-y^{m}}{1-y}\right) d y=\sum_{r=1}^{m} r^{-1} \sim \log m(m \rightarrow \infty) .
\end{aligned}
$$

Therefore

$$
\max _{1 \leq j \leq m}\left|J_{n, \varepsilon}\left(\omega_{j}\right)\right|^{2}=O_{,}(\log n) .
$$

Also, from (11),

$$
\left.\begin{array}{rlrl}
\left|Z_{n, u}(\omega)\right| & \leqq \sum_{v=1-u}^{0}\left|\varepsilon_{v}\right|+\sum_{v=n-u+1}^{n}\left|\varepsilon_{v}\right|, & u<n \\
& \leqq \sum_{v=1-u}^{n-u}\left|\varepsilon_{v}\right|+\sum_{v=1}^{n}\left|\varepsilon_{v}\right|, & & u \geqq n
\end{array}\right\},
$$

and, from (12),

$$
\max _{j}\left|R_{n}\left(\omega_{j}\right)\right| \leqq(2 / n)^{\frac{1}{2}} \sum_{u=1}^{\infty}\left|\alpha_{u}\right| \max _{j}\left|Z_{n, u}\left(\omega_{j}\right)\right| .
$$

Hence

$$
E\left\{\max _{j}\left|R_{n}\left(\omega_{j}\right)\right|\right\} \leqq(2 / n)^{\frac{1}{2}} 2 E\left(\left|\varepsilon_{t}\right|\right)\left\{\sum_{u=1}^{n-1} u\left|\alpha_{u}\right|+n \sum_{u=n}^{\infty}\left|\alpha_{u}\right|\right\},
$$

and so, since

$$
\begin{gathered}
\sum_{u=1}^{n-1} u\left|\alpha_{u}\right|<n^{\frac{1}{2}-\delta} \sum_{u=1}^{\infty} u^{\frac{1}{2+\delta}}\left|\alpha_{u}\right|, n \sum_{u=n}^{\infty}\left|\alpha_{u}\right| \leqq n^{\frac{1}{2}-\delta} \sum_{u=n}^{\infty} u^{\frac{1}{2}+\delta}\left|\alpha_{u}\right|, \\
E\left\{\max _{1 \leqq j \leqq m}\left|R_{n}\left(\omega_{j}\right)\right|\right\}=O\left(n^{-\delta}\right),
\end{gathered}
$$

giving

$$
\max _{1 \leqq j \leqq m}\left|R_{n}\left(\omega_{j}\right)\right|=O_{p}\left(n^{-\delta}\right) .
$$

The conclusion (18) then follows from (20), (22) and (26), since

$$
\max _{1 \leq j \leq m}\left|A\left(\omega_{j}\right)\right|<\sum_{u=0}^{\infty}\left|\alpha_{u}\right|<\infty \text {. }
$$

To obtain the corollary (19) we have only to apply Theorem 1 for $\omega=0, \pi$.

An alternative proof of Theorem 2, with (17) replaced by the slightly 
weaker condition $\sum_{u=1}^{\infty} u^{\frac{1}{2}}\left|\alpha_{u}\right|<\infty$, has been outlined by Hannan (1960, p. 79). This seems less straightforward than the proof we have just given, but yields a much better estimate of the order of magnitude of $\max _{1 \leqq j \leqq m}\left|T_{n}\left(\omega_{j}\right)\right|$ and also is easily modified so as to require only the condition $\sum_{u=1}^{\infty} u^{1+\delta}\left|\alpha_{u}\right|<\infty$ for some $\delta>0$. However, our proof can be strenghtened without much loss of simplicity so as to be valid under the latter condition, and then it shows that $\max _{1 \leqq j \leqq m}\left|T_{n}\left(\omega_{1}\right)\right|=O_{p}\left(n^{-\delta}\{\log n\}^{\frac{1}{2}}\right)$, which is only slightly weaker than the corresponding result that can be obtained with the modification of Hannan's method, namely $\max _{1 \leqq j \leqq m}\left|T_{n}\left(\omega_{j}\right)\right|=O_{p}\left(n^{-\delta}\right)$.

THEOREM $2 a$. Let the distribution of $\varepsilon_{t}$ be normal, and let

$$
\sum_{u=1}^{\infty} u^{1+\delta}\left|\alpha_{u}\right|<\infty \text {, for some } \delta>0 \text {. }
$$

Then the conclusion of Theorem 2 holds.

Proof. We replace (23) in the proof of Theorem 2 by the stricter inequalities

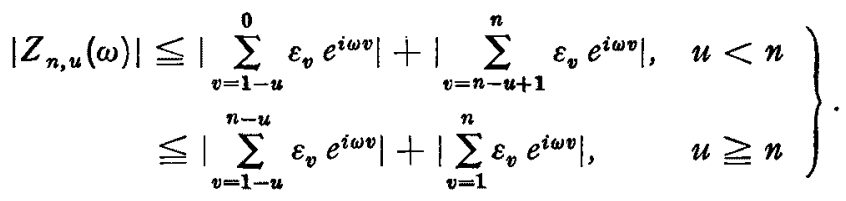

Now for any $r$,

$$
\left|\sum_{v=1}^{r} \varepsilon_{v} e^{i \omega v \mid}\right|^{2}=\sum_{s=-r}^{r} e^{i \omega s} \sum_{t=1}^{r-|s|} \varepsilon_{t} \varepsilon_{t+|s|} \leqq \sum_{s=-r}^{r}\left|\sum_{t=1}^{r-|s|} \varepsilon_{t} \varepsilon_{t+|s|}\right|
$$

Hence

$$
\begin{aligned}
E\left\{\max _{j}\left|\sum_{v=1}^{r} \varepsilon_{v} e^{i \omega, v}\right| 2\right\} & \leqq E\left(\sum_{t=1}^{r} \varepsilon_{t}^{2}\right)+2 \sum_{s=1}^{r-1}\left[E\left\{\left(\sum_{t=1}^{r-s} \varepsilon_{t} \varepsilon_{t+s}\right)^{2}\right\}\right]^{\frac{1}{2}} \\
& =r+2 \sum_{s=1}^{r-1}(r-s)^{\frac{1}{2}} \leqq r+2 \int_{1}^{r} x^{\frac{1}{2}} d x \\
& \leqq K r^{\frac{3}{2}}
\end{aligned}
$$

where $K$ is a constant independent of $r$, and so

$$
E\left\{\max _{j}\left|\sum_{v=1}^{r} \varepsilon_{v} e^{i \omega s v}\right|\right\} \leqq K^{\frac{1}{2}} r \frac{3}{*}
$$

(30) obviously still holds if the limits of summation with respect to $v$ are replaced by $1+p, r+p$, where $p$ is an arbitrary (positive or negative) integer. From (28) we therefore obtain

$$
E\left\{\max _{j}\left|Z_{n, u}\left(\omega_{j}\right)\right|\right\} \leqq 2 K^{\frac{1}{2}}\{\min (u, n)\}^{\frac{3}{4}},
$$


which gives in place of (25),

$$
\begin{aligned}
E\left\{\max _{j}\left|R_{n}\left(\omega_{s}\right)\right|\right\} & \leqq 2(2 K / n)^{\frac{1}{t}}\left\{\sum_{u=1}^{n-1} u^{z}\left|\alpha_{u}\right|+n^{\frac{3}{2}} \sum_{u=n}^{\infty}\left|\alpha_{u}\right|\right\} \\
& \leqq 2(2 K / n)^{\frac{1}{2}} n^{\frac{1}{2}-\delta}\left\{\sum_{u=1}^{n-1} u^{\frac{1+\delta}{4}}\left|\alpha_{u}\right|+\sum_{u=n}^{\infty} u^{\frac{1}{t+\delta}}\left|\alpha_{u}\right|\right\}
\end{aligned}
$$

Thus $E\left\{\max _{1 \leqq j \leqq m}\left|R_{n}\left(\omega_{j}\right)\right|\right\}=O\left(n^{-\delta}\right)$ and the remainder of the proof is as before.

It will be noticed that we have not made full use of $(28)$ in obtaining the upper bound (31) to $E\left\{\max _{1 \leqq j \leqq m}\left|Z_{n, u}\left(\omega_{j}\right)\right|\right\}$. In fact since

$$
\left|Z_{n, u}(\omega)\right|^{2} \leqq 2\left\{\left|\sum_{v=1-u}^{n-u} \varepsilon_{v} e^{i \omega v}\right|^{2}+\mid \sum_{v=1}^{n} \varepsilon_{v} e^{i \omega v \mid 2}\right\}
$$

follows from the second inequality, we can use (21) to give us the much sharper upper bound $2 \frac{3}{2}\{\log m+1\}^{\frac{1}{2}}$ for $u \geqq n$. This by itself is of no consequence since the first sum in (32) is $O\left(n^{-\delta}\right)$ and (as is easily seen) cannot in general be $O\left(n^{-\delta^{\prime}}\right)$ for $\delta^{\prime}>\delta$, but taken in conjunction with a sharper upper bound for $u<n$ should enable the theorem to be established under a condition on the $\left|\alpha_{u}\right|$ which is weaker than (27). We now show that such an upper bound can be obtained by an argument which is still fairly elementary.

LEMma 1. If $0 \leqq \omega \leqq \pi$,

$$
I_{n, \varepsilon}(\omega) \leqq K \log n \max _{0 \leqq r \leqq n} I_{n, \varepsilon}(r \pi / n)
$$

where $K$ is a suitable constant.

Proof. Writing $I_{n, \varepsilon}(\omega) \equiv 2 \phi(\omega)$ we have by definition,

$$
\phi(\omega)=\sum_{|s|<n} C_{s} e^{i \omega s}
$$

where $n C_{s}=\sum_{t=1}^{n-1 s]} \varepsilon_{t} \varepsilon_{i+s}$.

Hence, if we let $\omega_{r}$ for the moment denote $r \pi / n$ (not to be confused with its previous meaning),

$$
\begin{aligned}
& \sum_{r=-(n-1)}^{n} \phi\left(\omega_{r}\right) e^{i \omega_{r} s}=\sum_{\left|s^{\prime}\right|<n} C_{z^{\prime}} \sum_{r=-(n-1)}^{n} e^{i \omega_{r}\left(s^{\prime}-s\right)} \\
& \left.\begin{array}{lll}
=2 n C_{s}, & \text { if } & |s|<n \\
=0 & \text { if } & |s|=n
\end{array}\right\} .
\end{aligned}
$$

Therefore 


$$
\begin{aligned}
2 n \phi(\omega) & =\sum_{s=-(n-1)}^{n} e^{i \omega s} \sum_{r=-(n-1)}^{n} \phi\left(\omega_{r}\right) e^{-i \omega_{r} s} \\
& =\sum_{r=-(n-1)}^{n} \phi\left(\omega_{r}\right) e^{\frac{1}{2} i\left(\omega-\omega_{r}\right)}\left(\frac{\sin n\left(\omega-\omega_{r}\right)}{\sin \frac{1}{2}\left(\omega-\omega_{r}\right)}\right) \\
& =\sum_{r=-(n-1)}^{n} \phi\left(\omega_{r}\right) \cot \frac{1}{2}\left(\omega-\omega_{r}\right) \sin n\left(\omega-\omega_{r}\right)
\end{aligned}
$$

where if $\omega=\omega_{r}$ the product of the last two terms is to be replaced by its limit when $\omega$ tends to $\omega_{r}$, namely $2 n$.

Now let $\delta_{n}=\min _{0 \leqq r \leqq n} \max \left(0, \omega-\omega_{r}\right)$ be the least non-negative value of $\omega-\omega_{r}$ and suppose $\omega$ is not a multiple of $\pi / n$ so that $0<\delta_{n}<\pi / n$. Then $\left|\sin \frac{1}{2}\left(\omega-\omega_{r}\right)\right|$ takes the $2 n$ values $\sin \frac{1}{2} \delta_{n}, \sin \frac{1}{2}\left(\delta_{n}+\pi / n\right), \cdots$, $\sin \frac{1}{2}\left(\pi-\delta_{n}^{\prime}\right), \sin \frac{1}{2} \delta_{n}^{\prime}, \sin \frac{1}{2}\left(\delta_{n}^{\prime}+\pi / n\right), \cdots, \sin \frac{1}{2}\left(\pi-\delta_{n}\right)$, where $\delta_{n}^{\prime}=\pi / n-\delta_{n}$. Also

Hence

$$
\left|\sin n\left(\omega-\omega_{r}\right)\right|=\left|\sin n \delta_{n}\right|=\left|\sin n \delta_{n}^{\prime}\right| \text { for all } r
$$

$$
\sum_{r=-(n-1)}^{n}\left|\cot \frac{1}{2}\left(\omega-\omega_{r}\right) \sin n\left(\omega-\omega_{r}\right)\right|
$$

$$
\begin{aligned}
& \leqq\left|\sin n \delta_{n}\right| \sum_{r=0}^{n-1}\left\{\operatorname{cosec} \frac{1}{2}\left(\delta_{n}+\omega_{r}\right)+\operatorname{cosec} \frac{1}{2}\left(\delta_{n}^{\prime}+\omega_{r}\right)\right\} \\
& <\left|\sin n \delta_{n}\right|\left(\operatorname{cosec} \frac{1}{2} \delta_{n}+\operatorname{cosec} \frac{1}{2} \delta_{n}^{\prime}+\frac{2 n}{\pi}\left\{\int_{\delta_{n}}^{\pi / 2} \operatorname{cosec} x d x+\int_{\delta_{n}^{\prime}}^{\pi / 2} \operatorname{cosec} x d x\right\}\right)
\end{aligned}
$$

since $\operatorname{cosec} x$ is a decreasing function of $x$ for $0<x<\frac{1}{2} \pi$. Now

$$
\int_{\delta_{n}}^{\pi / 2} \operatorname{cosec} x d x=-\frac{1}{2} \log \tan \delta_{n}=-\frac{1}{2} \log \delta_{n}+O\left(n^{-2}\right) .
$$

Therefore $(35)$ is equal to

$$
\begin{aligned}
-(n / \pi) & \left|\sin n \delta_{n}\right|\left\{\log \delta_{n}+\log \delta_{n}^{\prime}\right\}+O(n) \\
= & -(n / \pi)\left\{\left|\sin n \delta_{n}\right| \log n \delta_{n}+\left|\sin n \delta_{n}^{\prime}\right| \log n \delta_{n}^{\prime}\right\}+O(n \log n) \\
= & O(n \log n)
\end{aligned}
$$

since the function $\sin x \log x$ is bounded for $0<x<\pi$. Hence, from (34),

$$
\begin{aligned}
2 n \phi(\omega) & \leqq \max _{0 \leqq r \leqq n} \phi\left(\omega_{r}\right) \sum_{r=-(n-1)}^{n}\left|\cot \frac{1}{2}\left(\omega-\omega_{r}\right) \sin n\left(\omega-\omega_{r}\right)\right| \\
& \leqq 2 K n \log n \max _{0 \leqq r \leq n} \phi\left(\omega_{r}\right)
\end{aligned}
$$

for all $n$ such that $\delta_{n}>0$, and this inequality holds trivially if $\delta_{n}=0$.

Now we have $E\left\{\max _{\text {even } r} I_{n, \varepsilon}(\gamma \pi / n)\right\}=O(\log n)$, using (21), and it is easy to show that the random variables $I_{n, \varepsilon}(r \pi / n)$ obtained by letting 
$r$ run through all odd values less than $n$ are mutually independent and each have probability density $e^{-x}$ (see, for example, Bartlett, 1955, p. 278, equation (12)), so that $E\left\{\max _{\text {odd } r} I_{n, \varepsilon}(r \pi / n)\right\}=O(\log n)$ also. From Lemma 1 we therefore obtain

$$
E\left\{\max _{0 \leqq \omega \leqq \pi} I_{n, \varepsilon}(\omega)\right\}=O\left(\{\log n\}^{2}\right)
$$

Hence, from the first inequality of (26),

$$
E\left\{\max _{1 \leq j \leq m}\left|Z_{n, u}\left(\omega_{j}\right)\right|\right\}=O\left(u^{\frac{1}{2}} \log u\right),(u \rightarrow \infty), u<n .
$$

THEOREM 2b. Let the distribution of $\varepsilon_{t}$ be normal, and let

$$
\sum_{u=1}^{\infty} u^{\delta}\left|\alpha_{u}\right|<\infty, \text { for some } \delta>0
$$

Then the conclusion of Theorem 2 holds.

Proof. From (24) and (37),

$$
E\left\{\max _{1 \leqq j \leqq m}\left|R_{n}\left(\omega_{j}\right)\right|\right\} \leqq K n^{-\frac{1}{2}}\left\{\sum_{u=1}^{n-1} u^{\frac{1}{2}}(\log u)\left|\alpha_{u}\right|+(n \log n)^{\frac{1}{2}} \sum_{u=n}^{\infty}\left|\alpha_{u}\right|\right\}
$$

(where $K$ is a suitable constant)

$$
\begin{aligned}
& \leqq K n^{-\delta}\left\{(\log n) \sum_{u=1}^{n-1} u^{\delta}\left|\alpha_{u}\right|+(\log n)^{\frac{1}{2}} \sum_{u=n}^{\infty} u^{\delta}\left|\alpha_{u}\right|\right\} \\
& =O\left(n^{-\delta^{\prime}}\right) \text { for any } \delta^{\prime}<\delta
\end{aligned}
$$

The conclusion then follows as before.

Now suppose that the distribution of $\varepsilon_{t}$ is arbitrary. In this case the restriction of $\omega$ to the set $\left\{\omega_{i}\right\}$ does not simplify matters and we therefore consider the behaviour of $\max _{0 \leqq \omega \leqq \pi}\left|T_{n}(\omega)\right|$.

Theorem 3. Let

$$
\sum_{u=1}^{\infty} u^{\frac{1}{2}}\left|\alpha_{u}\right|<\infty
$$

Then

$$
p \lim _{n \rightarrow \infty}\left\{\max _{0 \leqq \omega ミ \pi}\left|T_{n}(\omega)\right|\right\}=0 .
$$

Proof. By the argument used to derive (32) (in the proof of Theorem 2a), which does not require the distribution of $\varepsilon_{t}$ to be normal,

$$
\begin{aligned}
E\left\{\max _{0 \leqq \omega \leqq \pi}\left|R_{n}(\omega)\right|\right\} & \leqq 2(2 K / n)^{\frac{1}{2}}\left\{\sum_{u=1}^{n-1} u u^{\frac{3}{k}}\left|\alpha_{u}\right|+n^{\frac{3}{3}} \sum_{u=n}^{\infty}\left|\alpha_{u}\right|\right\} \\
& \leqq 2(2 K) n^{-\frac{1}{4}}\left\{\sum_{u=1}^{n-1} u^{\frac{1}{2}}\left|\alpha_{u}\right|(u / n)^{\frac{1}{2}}+\sum_{u=n}^{\infty} u^{\frac{1}{2}}\left|\alpha_{u}\right|\right\} \\
& =o\left(n^{-1}\right),
\end{aligned}
$$


since the first sum is not greater than

$$
\sum_{u=k(n)+1}^{n-1} u \frac{1}{2}\left|\alpha_{u}\right|+\{k(n) / n\} \sum_{u=1}^{k(n)} u \frac{1}{2}\left|\alpha_{u}\right|,
$$

where $k(n)$ (as in the argument leading to (15)) is such that $\lim _{n \rightarrow \infty}\{k(n) / n\}=0$, $\lim _{n \rightarrow \infty} k(n)=\infty$.

Also by the argument used to derive (27),

$$
E\left\{\max _{0 \leqq \omega \leqq \pi}\left|\sum_{v=1}^{n} \varepsilon_{v} e^{i \omega v}\right|^{2}\right\} \leqq K n^{\frac{3}{2}}
$$

The result (41) then follows from

$$
\begin{aligned}
\max _{\omega}\left|T_{n}(\omega)\right| \leqq & 2 \max _{\omega}\left|R_{n}(\omega)\right| \max _{\omega}|A(\omega)| \max _{\omega}\left|J_{n, \varepsilon}(\omega)\right| \\
& +\left\{\max _{\omega}\left|R_{n}(\omega)\right|\right\}^{2} .
\end{aligned}
$$

Again it is possible to weaken the condition on the $\alpha_{u}$ at the expense of some complication. Provided that $E\left(\varepsilon_{t}^{8}\right)<\infty$, Hannan's method is still applicable and gives $\max _{1 \leq j \leq m}\left|T_{n}(2 \pi j \mid n)\right|=O_{p}\left(n^{-\delta}\right)$ under the condition (27) of Theorem 2a. It is not difficult to see that (41) can be deduced from this and the corresponding result in which the argument of $T_{n}$ ranges over odd multiples of $\pi / n$, by using the relation obtained by replacing $I_{n, \varepsilon}(\omega)$ by $\left|T_{n}(\omega)\right|$ in Lemma 1 .

Alternatively, we can make use of a remarkable result due to Whittle (1959, p. 180, equation (44)), which, in our notation, states that

provided that

$$
p \lim _{n \rightarrow \infty}\left\{\max _{0 \leqq \omega \leqq \pi} I_{n, \varepsilon}(\omega) / 2 \log n\right\}=1,
$$

$$
E\left(\left|\varepsilon_{t}\right|^{r}\right)<\infty \text { for some } r>4 .
$$

The arguments required to establish this are incidentally much more sophisticated, depending on one quite deep probability theorem of the same type as a theorem of Cramér concerning asymptotic expansions for distribution functions of sums of independent random variables (Cramér, 1962, p. 85, Theorem 26), and also a theorem of Bernstein (Zygmund, 1959, p. 11), stating that for any trigonometric polynomial $T(\omega)=\sum_{|k|<d} c_{k} e^{i k \omega}$ of degree $d, \max _{\omega}\left|T^{\prime}(\omega)\right|<d \max _{\omega}|T(\omega)|$, which, although fairly elementary in character, is probably familiar only to someone who has made a specialised study of the theory of trigonometric series. The proof given by Whittle is decidedly sketchy in places and suggests that (44) ought to be strengthened to

$$
E\left(\left|\varepsilon_{l}\right|^{*}\right)<\infty \text { for some } r>6
$$


however (44) certainly gives the weaker result

$$
\left.\lim _{n \rightarrow \infty} p \underset{0 \leqq \omega \leqq \pi}{\max } I_{n, \varepsilon}(\omega) \leqq 2 \log n(1+\eta)\right\}=1, \text { for any } \eta>0,
$$

which is sufficient for our purpose.

THEOREM 3a. Let $E\left(\left|\varepsilon_{t}\right|^{r}\right)<\infty$ for some $r>4$, and let

$$
\sum_{u=1}^{\infty} u^{1+\delta}\left|\alpha_{u}\right|<\infty
$$

Then the conclusion of Theorem 3 holds.

Proof. Proceeding as in the proof of Theorem 2a, we have

$$
E\left\{\max _{0 \leqq \omega \leqq \pi}\left|R_{n}(\omega)\right|\right\}=O\left(n^{-\delta}\right) .
$$

Hence, from (45),

$$
\max _{0 \leqq \omega \leqq \pi}\left|R_{n}(\omega)\right| \max _{0 \leqq \omega \leqq \pi}\left|J_{n, \varepsilon}(\omega)\right|=O_{p}\left\{n^{-\delta}(\log n)^{\frac{1}{2}}\right\},
$$

amd the conclusion then follows from (20a).

It might be thought that the result (45) would enable one to obtain a much stronger probability order of magnitude statement which would allow the condition on the $\alpha_{u}$ to be weakened still further. However, while this clearly gives us (from (28))

$$
\left.\begin{array}{rl}
\max _{0 \leqq \omega \leqq \pi}\left|Z_{n, u}(\omega)\right|^{2} & =O_{p}(u \log u), \quad u<n \\
& =O_{p}(n \log n), \quad u \geqq n
\end{array}\right\},
$$

it does not tell us anything about the behaviour of

$E\left\{\max _{0 \leqq \omega \leqq \pi}\left|Z_{n, u}(\omega)\right|^{2}\right\}$, and there seems to be no way of using (46)

directly because a countably infinite set of random variables $\max _{\omega}\left|Z_{n, u}(\omega)\right|$ appears in the upper bound to $\max _{\omega}\left|R_{n}(\omega)\right|$ corresponding to (24). For normal $\varepsilon_{t}$, it is easy to see that an argument almost identical to that used in the proof of Theorem $2 b$ will give us the following result.

THEOREM $3 b$. Let the distribution of $\varepsilon_{t}$ be normal, and let

$$
\sum_{u=1}^{\infty} u^{\delta}\left|\alpha_{u}\right|<\infty, \text { for some } \delta>0 \text {. }
$$

Then the conclusion of Theorem 3 holds. 


\section{The asymptotic distribution of a finite set of periodogram values} value.

We first obtain the asymptotic distribution of a single periodogram

THEOREM 4. Let $f(\omega) \neq 0$. Then when $n \rightarrow \infty$, the distribution of $I_{n, x}(\omega)$ tends to that of $2 \pi f(\omega) \chi_{2}^{2}$ if $0<\omega<\pi$ or $2 \pi f(\omega) \chi_{1}^{2}$ if $\omega=0$ or $\pi$, where the symbol $\chi_{r}^{2}$ denotes a random variable having the standard $\chi^{2}$ distribution with $r$ degrees of freedom.

Proof. Suppose first that $0<\omega<\pi$. Then the joint distribution of $\mathscr{R}\left\{J_{n, \varepsilon}(\omega)\right\}=(2 / n)^{\frac{1}{2}} \sum_{t=1}^{n} \varepsilon_{t} \cos \omega t$ and $\mathscr{I}\left\{J_{n, \varepsilon}(\omega)\right\}=(2 / n)^{\frac{1}{2}} \sum_{t=1}^{n} \varepsilon_{t}$ sin $\omega t$ tends to that of two independent random variables each distributed as $N(0,1)$. For let $U_{n}=\sum_{t=1}^{n} \eta_{t}$, where $\eta_{t}=\varepsilon_{t}(a \cos \omega t+b \sin \omega t), a, b$ being arbitrary real constants. Then the sequence $\left\{\eta_{t}\right\}, t=1,2,3, \cdots$, obeys the Lindeberg condition for the validity of the Central Limit Theorem, namely

$$
\lim _{n \rightarrow \infty}\left(\sum_{t=1}^{n} \int_{|\eta|>\delta \sigma_{n}} \eta^{2} d G_{t}(\eta) / \sigma_{n}^{2}\right)=0 \text { for each } \delta>0,
$$

where $\sigma_{n}^{2}=E\left(U_{n}^{2}\right)$ and $G_{t}$ is the distribution function of $\eta_{t}$ (see, for example, Cramér, 1962, p. 58). For the left-hand side of (47) is equal to

$$
\sigma_{n}^{-2} \sum_{t=1}^{n} c_{t}^{2} \int_{|\varepsilon|>\delta \sigma_{n}|| c_{t} \mid} \varepsilon^{2} d F(\varepsilon)
$$

where $F$ is the distribution function of $\varepsilon_{t}$, and $c_{t}=a \cos \omega t+b \sin \omega t$. Now $c_{t}^{2} \leqq a^{2}+b^{2}$ for all $t$ and $\sum_{t=1}^{n} c_{t}^{2}=\sigma_{n}^{2}$. Hence (48) does not exceed $\int_{|\varepsilon|>\delta \sigma_{n} /\left(a^{2}+b^{2}\right)^{\frac{1}{2}}} \varepsilon^{2} d F(\varepsilon)$, which tends to zero as $n \rightarrow \infty$ since $\sigma_{n}^{2} \sim \frac{1}{2} n\left(a^{2}+b^{2}\right)$. The limiting distribution of $U_{n} / \sigma_{n}$ is therefore $N(0,1)$, so that the limiting distribution of $(2 / n)^{\frac{1}{2}} U_{n}$ is $N\left(0, a^{2}+b^{2}\right)$. The result follows from this by applying the continuity theorem for characteristic functions to deduce that

$$
\lim _{n \rightarrow \infty} E\left[\exp \left(i \theta_{1} \mathscr{R}\left\{J_{n, \varepsilon}(\omega)\right\}+i \theta_{2} \mathscr{I}\left\{J_{n, \varepsilon}(\omega)\right\}\right)\right]=\exp -\frac{1}{2}\left(\theta_{1}^{2}+\theta_{2}^{2}\right)
$$

for arbitrary real $\theta_{1}, \theta_{2}$.

From the conclusion (9) of Theorem 1 we therefore see that the limiting joint distribution of $\mathscr{R}\left\{J_{n, x}(\omega)\right\}, \mathscr{I}\left\{J_{n, x}(\omega)\right\}$ is the same as that of

and

$$
\mathscr{R}\{A(\omega)\} \mathscr{R}\left\{J_{n, \varepsilon}(\omega)\right\}-\mathscr{I}\{A(\omega)\} \mathscr{I}\left\{J_{n, \varepsilon}(\omega)\right\}
$$

$$
\mathscr{I}\{A(\omega)\} \mathscr{R}\left\{J_{n, \varepsilon}(\omega)\right\}+\mathscr{R}\{A(\omega)\} \mathscr{I}\left\{J_{n, \varepsilon}(\omega)\right\}
$$

respectively, namely that of two independent random variables each distributed as $N\left(0,|A(\omega)|{ }^{2}\right)$. Hence the limiting distribution of $I_{n, x}(\omega)$ is that of $|A(\omega)|^{2} \chi_{2}^{2}$, and, according to $(4),|A(\omega)|^{2}=2 \pi f(\omega)$. Alternatively 
(and this is slightly simpler) the limiting distribution of $I_{n, \varepsilon}(\omega)$ is that of $\chi_{2}^{2}$ and the conclusion of the theorem then follows from (10).

If $\omega=0$ or $\pi$ a similar argument applies, the number of degrees of freedom of $\chi^{2}$ being reduced from 2 to 1 since the imaginary parts of $J_{n, x}(\omega)$ and $J_{n, \varepsilon}(\omega)$ become identically zero.

Suppose now that $\left\{\omega_{r}\right\}, 1 \leqq r \leqq k$ is a finite set of fixed angular frequencies. Then the asymptotic distribution of the set of periodogram values $\left\{I_{n, x}\left(\omega_{r}\right)\right\}$ is given by the following theorem.

THEOREM 5. Let $f\left(\omega_{r}\right) \neq 0,1 \leqq r \leqq k$. Then when $n \rightarrow \infty$ the joint distribution of $I_{n, x}\left(\omega_{r}\right), 1 \leqq r \leqq k$, tends to that of $k$ mutually independent random variables, distributed as $2 \pi f\left(\omega_{r}\right) \chi_{2}^{2}$ if $0<\omega_{r}<\pi$ and $2 \pi f\left(\omega_{r}\right) \chi_{1}^{2}$ if $\omega_{r}=0$ or $\pi$.

Proof. Let $U_{n}=\sum_{t=1}^{n} c_{t} \varepsilon_{t}$, where $c_{t}=\sum_{r=1}^{k}\left(a_{r} \cos \omega_{r} t+b_{r} \sin \omega_{r} t\right), a_{r}, b_{r}$ being arbitrary real constants, and suppose that $0<\omega_{r}<\pi(1 \leqq r \leqq k)$. Then an argument similar to that used in the proof of Theorem 4 shows that the limiting distribution of $(2 / n)^{\frac{1}{2}} U_{n}$ is $N\left(0, \sum_{r=1}^{k}\left\{a_{r}^{2}+b_{r}^{2}\right\}\right)$, and hence that the limiting joint distribution of $\mathscr{R}\left\{J_{n, \varepsilon}\left(\omega_{r}\right)\right\}, \mathscr{I}\left\{J_{n, \varepsilon}\left(\omega_{r}\right)\right\}(1 \leqq r \leqq k)$ is that of $2 k$ mutually independent random variables each distributed as $N(0,1)$. The limiting joint distribution of $I_{n, \varepsilon}\left(\omega_{r}\right)(1 \leqq r \leqq k)$ is therefore that of $k$ mutually independent random variables each distributed as $\chi_{2}^{2}$, and the conclusion follows from (10). The only modification required if $\omega_{r}=0$ or $\pi$ for some $r$ is clearly the reduction of the number of degrees of freedom of the corresponding $\chi^{2}$ by 1 .

\section{The asymptotic distribution of the largest periodogram value for an angular frequency which is a multiple of $2 \pi / n$}

We now suppose that periodogram values $I_{n, x}\left(\omega_{j}\right)$ have been computed for all angular frequencies $\omega_{j}=2 \pi j / n$, where $j$ is an integer, and consider the asymptotic behaviour of

(i) the largest of the $I_{n, x}\left(\omega_{j}\right)$,

(ii) the largest of the quantities $K_{n, x}\left(\omega_{j}\right)=I_{n, x}\left(\omega_{j}\right) /\left\{2 \pi f\left(\omega_{j}\right)\right\}$, namely the periodogram values 'standardised' by division by factors $2 \pi f\left(\omega_{j}\right)=$ $\left|A\left(\omega_{j}\right)\right|^{2}$, which (from (10)) make them approximately equal to the corresponding values of the periodogram of the residual process $\left\{\varepsilon_{t}\right\}$ when $n$ is large. (ii) presents the simpler problem and we therefore deal with this first.

THEOREM 6. Let the distribution of $\varepsilon_{t}$ be normal, the sequence $\left\{\alpha_{u}\right\}$ satisfy one of the conditions in Theorems $2,2 a, 2 b$ (the weakest of which is that in Theorem $2 b$, namely $\left.\sum_{u=1}^{\infty} u^{\delta}\left|\alpha_{u}\right|<\infty\right)$, and let $f(w)>0,0 \leqq \omega \leqq \pi$. Then 
the asymptotic distribution of $\max _{1 \leqq j \leqq m} K_{n, x}\left(\omega_{j}\right)$, where $m=[(n-1) / 2]$, is given by

(49) $\lim _{n \rightarrow \infty} p\left\{\frac{1}{2} \max _{1 \leq j \leq m} K_{n, x}\left(\omega_{j}\right)-\log m>u\right\}=1-\exp \left(-e^{-u}\right)$ for all fixed $u>0$.

COROLLARY 1. In (49) $\max _{1 \leqq j \leqq m} K_{n, x}\left(\omega_{j}\right)$ may be replaced by

$$
\max _{0 \leqq i \leqq[n / 2]} K_{n, \infty}\left(\omega_{j}\right) \text {. }
$$

COROLLARY 2. In (49) $\frac{1}{2} \max _{1 \leqq j \leqq m} K_{n, x}\left(\omega_{j}\right)$ may be replaced by $\max _{1 \leqq j \leq m} K_{n, x}\left(\omega_{j}\right) / m^{-1} \sum_{j=1}^{m} K_{n, x}\left(\omega_{j}\right)$ or $\max _{0 \leqq i \leqq[n / 2]} K_{n, x}\left(\omega_{j}\right) /([n / 2])^{-1} \sum_{j=0}^{[n / 2]} K_{n, x}\left(\omega_{j}\right)$.

Proof. We have

$$
\max _{1 \leqq j \leqq m}\left|K_{n, x}\left(\omega_{i}\right)-I_{n, \varepsilon}\left(\omega_{j}\right)\right| \leqq \max _{1 \leqq j \leqq m}\left\{2 \pi f\left(\omega_{j}\right)\right\}^{-1} \max _{1 \leqq j \leqq m}\left|I_{n, z}\left(\omega_{j}\right)-2 \pi f\left(\omega_{j}\right) I_{n, \varepsilon}\left(\omega_{j}\right)\right|,
$$

and $f(\omega)$ is a continuous function, so that it must have a positive lower bound, and therefore $\max _{1 \leqq j \leqq m}\left\{2 \pi f\left(\omega_{j}\right)\right\}^{-1}$ has a finite upper bound. Hence from Theorem 2, $2 a$, or $2 \mathrm{~b}$,

Since

$$
p \lim _{n \rightarrow \infty} \max _{1 \leqq j \leqq m}\left|K_{n, x}\left(\omega_{j}\right)-I_{n, e}\left(\omega_{j}\right)\right|=0 .
$$

$$
I_{n, \varepsilon}\left(\omega_{j}\right)-\left|K_{n, x}\left(\omega_{j}\right)-I_{n, \varepsilon}\left(\omega_{j}\right)\right| \leqq K_{n, x}\left(\omega_{j}\right) \leqq I_{n, \varepsilon}\left(\omega_{j}\right)+\left|K_{n, x}\left(\omega_{j}\right)-I_{n, \varepsilon}\left(\omega_{j}\right)\right|,
$$

it follows that

$$
p \lim _{n \rightarrow \infty}\left|\max _{1 \leqq j \leqq m} K_{n, x}\left(\omega_{j}\right)-\max _{1 \leq i \leq m} I_{n, \varepsilon}\left(\omega_{j}\right)\right|=0 .
$$

Also (as we noted in the proof of Theorem 2)

Hence

$$
p\left\{\frac{1}{2} \max _{1 \leqq j \leqq m} I_{n, \varepsilon}\left(\omega_{j}\right)<x\right\}=\left(1-e^{-x}\right)^{m} .
$$

$$
\begin{aligned}
\lim _{n \rightarrow \infty} p\left\{\frac{1}{2} \max _{1 \leqq j \leqq m} I_{n, \varepsilon}\left(\omega_{j}\right)\right. & >u+\log m\}=\lim _{n \rightarrow \infty}\left\{1-\left(1-e^{-(u+\log m)}\right)^{m}\right\} \\
& =\lim _{m \rightarrow \infty}\left\{1-\left(1-m^{-1} e^{-u}\right)^{m}\right\}=1-\exp \left(-e^{-u}\right) .
\end{aligned}
$$

From (50) and (51) we therefore obtain (49).

Corollary 1 is an immediate consequence of the fact that $K_{n, x}(0)$, $K_{n, x}(\pi)$ are each $O_{\nu}(1)$, by Theorem 4 . To establish the first part of Corollary 2 we write

$$
m^{-1} \sum_{j=1}^{m} K_{n, x}\left(\omega_{j}\right)=m^{-1} \sum_{j=1}^{m} I_{n, \varepsilon}\left(\omega_{j}\right)+m^{-1} \sum_{j=1}^{m}\left[T_{n}\left(\omega_{i}\right) /\left\{2 \pi f\left(\omega_{j}\right)\right\}\right]
$$


$T_{n}(\omega)$ being defined by (8). The modulus of the second term on the righthand side of (52) does not exceed $\left.\max _{1 \leq j \leq m}\left\{2 \pi f\left(\omega_{j}\right)\right\}^{-1} \max _{1 \leq j \leq m} \mid T_{n}\left(\omega_{j}\right)\right)$, and therefore, from the final result yielding the conclusion of Theorem 2, is $O_{p}\left(n^{-\delta^{\prime}}\right)$ for some $\delta^{\prime}>0$. Also $\sum_{j=1}^{m} I_{n, \varepsilon}\left(\omega_{j}\right)$ is distributed as $\chi_{2 m}^{2}$, and hence

$$
m^{-1} \sum_{j=1}^{m} K_{n, x}\left(\omega_{j}\right)=2+O_{p}\left(n^{-\delta^{\prime}}\right),
$$

if we take $\delta^{\prime}<\frac{1}{2}$.

It follows that

$$
p \lim _{n \rightarrow \infty} \max _{1 \leqq j \leqq m} K_{n, x}\left(\omega_{j}\right)\left\{1-\left(2 / m^{-1} \sum_{j=1}^{m} K_{n, x}\left(\omega_{j}\right)\right)\right\}=0,
$$

which establishes the result. The second part of Corollary 2 is obtained in exactly the same way.

The above proof is fairly closely related to one given by Hannan (1960, pp. 79-80). However, he does not specify the precise form of the asymptotic distribution, and there also seems to be an error in his argument at one point.

The main application of Theorem 6 is the construction of large-sample tests for detecting a jump in the spectral distribution function of $\left\{x_{t}\right\}$, with $x_{t}=\sum_{u=0}^{\infty} \alpha_{u} \varepsilon_{t-u}$ under the null hypothesis and $x_{t}=\sum_{u=0}^{\infty} \alpha_{u} \varepsilon_{t-u}+$ $A \cos \omega t+B \sin \omega t$ under the alternative hypothesis, where $A, B, \omega$ are unknown parameters. These were first proposed by Whittle (1952, p. 47, 1954, p. 222). Situations in which, under the null hypothesis, $f(\omega)$ is completely specified, or specified to within a factor of proportionality, that is, $f(\omega)=c g(\omega)$, where $g$ is a known function, so that the statistics in Corollary 2 can still be computed, are fairly rare, but when $f$ depends on a finite set of parameters it will usually be permissible to apply the theorem when $K_{n, x}\left(\omega_{j}\right)$ is replaced by $\hat{K}_{n, x}\left(\omega_{j}\right)=I_{n, x}\left(\omega_{j}\right) /\left\{2 \pi \hat{f}\left(\omega_{j}\right)\right\}$, where $\hat{f}$ denotes the estimated spectral density function obtained by replacing the parameters by suitable estimates. For example, if $h=g^{-1}$ depends on a set of parameters $\theta=\left(\theta_{1}, \theta_{2}, \cdots \theta_{p}\right)$, the partial derivatives $\partial h / \partial \theta_{i}(1 \leqq i \leqq p)$ are bounded for $0 \leqq \omega \leqq \pi$, and estimates $\hat{\theta}=\left(\hat{\theta}_{1}, \hat{\theta}_{2}, \cdots \hat{\theta}_{\boldsymbol{v}}\right)$ exist such that $\hat{\theta}_{i}-\theta_{i}=O_{p}\left(n^{\left.-\frac{1}{2}\right)}\right.$, it is easy to see that this follows because we have $\max _{j}\left|h\left(\omega_{j}, \boldsymbol{\theta}\right)-h\left(\omega_{j}, \hat{\theta}\right)\right|=O_{p}\left(n^{-\frac{1}{2}}\right)$. In particular, these conditions are satisfied when $\left\{x_{t}\right\}$ is a stationary autoregressive process such that $x_{t}+\sum_{r=1}^{p} \theta_{r} x_{t-r}=c^{\frac{1}{2}} \varepsilon_{t}$, so that $h(\omega, \theta)=\left|1+\sum_{r=1}^{p} \theta_{r} e^{i \omega r}\right|^{2} \quad$ (compare Hannan, 1960, p. 81). Estimates $\hat{f}\left(\omega_{j}\right)$ obtained by non-parametric methods can also be used (Hannan, 1961, pp. 400-1).

In connection with this application it should be noted that if we put $1-\exp \left(-e^{-u}\right)=P, u=-\log \{-\log (1-P)\}$, which is very nearly equal to $-\log P$ when $P$ is small. The upper significance point of 
$\frac{1}{2} \max _{1 \leqq j \leqq m} K_{n, x}\left(\omega_{j}\right)$ for level $P$ will therefore be approximately $\log (n / 2 P)$ (compare Priestley, 1962, p. 515, equation (2.3)).

If the distribution of $\varepsilon_{t}$ is not normal the conclusion of Theorem 6 will clearly still hold when $\left\{\alpha_{u}\right\}$ satisfies the condition of Theorem 3 (or Theorem 3a when $E\left(\left|\varepsilon_{t}\right|^{r}\right)<\infty$ for some $r>4$ ) provided that (51) can be established. It seems reasonable to expect (51) to hold provided that the moments of the distribution of $\varepsilon_{t}$ up to some sufficiently high order exist, in view of Theorem 5 and the fact that $\operatorname{cov}\left\{I_{n, \varepsilon}\left(\omega_{j}\right), I_{n, \varepsilon}\left(\omega_{k}\right)\right\}=O\left(n^{-1}\right)$ for $j \neq k$ (see, for example, Hannan 1960, p. 53), but no proof is known, and the problem of constructing one is undoubtedly extremely difficult. However, arguments used by Whittle (1959, pp. 176-80) suggest that the following result can be proved rigorously.

Then if

LEMMA 2. Let $p_{n}(P)=p\left\{\frac{1}{2} \max _{1 \leqq j \leqq m} I_{n, \varepsilon}\left(\omega_{j}\right)>\log (m / P)\right\}$.

$$
\begin{gathered}
E\left\{\left|\varepsilon_{t}\right|^{r}\right\}<\infty \text { for some } r>6, \\
P-\frac{1}{2} P^{2} \leqq \varliminf_{n \rightarrow \infty} p_{n}(P), \varlimsup_{n \rightarrow \infty} p_{n}(P) \leqq P,
\end{gathered}
$$

so that, for sufficiently large $n$,

$$
P-\frac{1}{2} P^{2} \leqq p_{n}(P) \leqq P .
$$

For if we write $Y_{j}=\frac{1}{2} I_{n, \varepsilon}\left(\omega_{j}\right), c_{n}=\log (m / P)$, we have

$$
\sum_{j=1}^{m} p\left(Y_{j}>c_{n}\right)-\sum_{j>k=1}^{m} p\left(Y_{j}>c_{n}, Y_{k}>c_{n}\right) \leqq p_{n}(P) \leqq \sum_{j=1}^{m} p\left(Y_{j}>c_{n}\right),
$$

since for any set of events $\left\{A_{j}\right\}, 1 \leqq j \leqq m$,

$$
\sum_{j=1}^{m} p\left(A_{j}\right)-\sum_{j>k=1}^{m} p\left(A_{j} \cap A_{k}\right) \leqq p\left(\bigcup_{j=1}^{m} A_{j}\right) \leqq \sum_{j=1}^{m} p\left(A_{j}\right) .
$$

(See, for example, Feller, 1957, p. 100, equation (5.7)).

Now an asymptotic expansion of the joint distribution function of $(2 / n)^{\frac{1}{2}} \sum_{t=1}^{n} \varepsilon_{t} \cos \omega_{j} t$ and $(2 / n)^{\frac{1}{2}} \sum_{t=1}^{n} \varepsilon_{t} \sin \omega_{j} t$, obtained by expanding the cumulant-generating function up to terms involving joint cumulants of order $r$, will give

$$
p\left(Y_{j}>c_{n}\right)=e^{-c_{n}}\left\{1+K_{1}\left(n, c_{n}, \omega_{j}\right)\right\}+R_{1}\left(n, c_{n}, \omega_{j}\right)
$$

where, when $n \rightarrow \infty, K_{1}$ tends to zero and $R_{1}$ is $0\left(n^{-\frac{1}{2}(r-2)}\right)$, both uniformly in $j$. (Compare Whittle, 1959, equation (43), which however is not completely accurate). Also from a similar expansion of the joint distribution function of $(2 / n)^{\frac{1}{2}} \sum_{t=1}^{n} \varepsilon_{t} \cos \omega_{j} t,(2 / n)^{\frac{1}{2}} \sum_{t=1}^{n} \varepsilon_{t} \sin \omega_{j} t,(2 / n)^{\frac{1}{2}} \sum_{t=1}^{n} \varepsilon_{t} \cos \omega_{k} t$, $(2 / n)^{\frac{1}{2}} \sum_{t=1}^{n} \varepsilon_{t} \sin \omega_{k} t$ we obtain 


$$
p\left(Y_{j}>c_{n}, Y_{k}>c_{n}\right)=e^{-2 c_{n}}\left\{1+K_{2}\left(n, c_{n}, \omega_{j}\right)\right\}+R_{2}\left(n, c_{n}, \omega_{j}\right)
$$

where, when $n \rightarrow \infty, K_{2}$ tends to zero and $R_{2}$ is $0\left(n^{-\frac{1}{2}(r-2)}\right)$, again both uniformly in $j$.

From (55), we see that

$$
\lim _{n \rightarrow \infty} \sum_{j=1}^{m} p\left(Y_{j}>c_{n}\right)=m e^{-c_{n}}=P,
$$

and similarly from (56),

$$
\lim _{n \rightarrow \infty} \sum_{j>k=1}^{m} p\left(Y_{j}>c_{n}, Y_{k}>c_{n}\right)=\lim _{m \rightarrow \infty} \frac{1}{2} m(m-1) e^{-2 c_{n}}=\frac{1}{2} P^{2}
$$

(53) then follows on using (54).

As an immediate consequence of Lemma 2 and the argument used in the proof of Theorem 6 we have the following result.

THEOREM 7. Let $E\left\{\left|\varepsilon_{t}\right|^{r}\right\}$ for some $r>6,\left\{\alpha_{u}\right\}$ satisfy one of the conditions in Theorems $2,2 a, 2 b$, and let $f(\omega)>0,0 \leqq \omega \leqq \pi$. Then for sufficiently large $n$,

$$
P-\frac{1}{2} P^{2} \leqq p\left\{\frac{1}{2} \max _{1 \leqq j \leqq m} K_{n, x}\left(\omega_{j}\right)>\log (m / P)\right\} \leqq P .
$$

In applications we are usually interested in small values of $P$ (say $P \leqq 0.1)$ and then to a very good approximation

$$
p\left\{\frac{1}{2} \max _{1 \leqq j \leqq m} K_{n, x}\left(\omega_{j}\right)>\log (m / P)\right\}=P .
$$

It is easy to see that the analogues of Corollaries 1 and 2 of Theorem 6 apply to $(59)$ and $(60)$.

Passing now to the problem (i) we have the following result corresponding to Theorem 6 .

THEOREM 8. Let the conditions of Theorem 6 be satisfied, and also let the spectral density thave a unique maximum at $\omega=\omega_{0} \neq 0$ or $\pi$, in some neighbourhood of which its second derivative is continuous. Then if $c_{n}$ is defined by

$$
g\left(\omega_{0}\right) c_{n}=\log m-\frac{1}{2} \log \log m+\frac{1}{2} \log \left\{2 g\left(\omega_{0}\right) / \pi g^{\prime \prime}\left(\omega_{0}\right)\right\}-\log \{-\log (1-P)\}
$$

where $g(\omega)=\{2 \pi f(\omega)\}^{-1}$,

$$
\lim _{n \rightarrow \infty} p\left\{\frac{1}{2} \max _{1 \leqq j \leqq m} I_{n, x}\left(\omega_{j}\right)>c_{n}\right\}=P .
$$

Proof. We employ an argument which is a more rigorous version of that given by Walker (1964), who required the result in connection with the problem of testing a hypothesis of the form $x_{t}=\sum_{u=0}^{\infty} \alpha_{u} \varepsilon_{t-u}$ against 
alternatives or the form $x_{t}=\varepsilon_{t}+A \cos \omega t+B \sin \omega t$. We have, writing $g_{j}=g\left(\omega_{j}\right)$,

(63) $-\log p\left\{\frac{1}{2} I_{n, \varepsilon}\left(\omega_{j}\right) \leqq c_{n} g_{j}, 1 \leqq j \leqq m\right\}=-\sum_{j=1}^{m} \log \left(1-e^{-c_{n} \theta_{j}}\right) \sim \sum_{j=1}^{m} e^{-c_{n} \theta_{s}}$,

provided that (63) tends to a positive limit when $n \rightarrow \infty$. Now, using the simplest case of the Euler-Maclaurin sum formula (Cramér, 1946, p. 124, equation (12.2.3)), we obtain

$$
\sum_{j=1}^{m} e^{-c_{n} g_{j}}=(n / 2 \pi) \int_{0}^{\pi} e^{-c_{n} g(\omega)} d \omega+\left\{2 \pi c_{n} g^{\prime}(\omega) / n\right\} \int_{0}^{\pi} P_{1}\left(\frac{n \omega}{2 \pi}\right) e^{-c_{n} g(\omega)} d \omega+o(1)
$$

where $P_{1}(x)=[x]-x+\frac{1}{2}$ for all non-integral $x$.

The second term on the right-hand side of (64) clearly tends to zero when $n \rightarrow \infty$ since the integral occurring in it has a finite upper bound and $c_{n}=O(\log n)$. Hence

$$
\sum_{j=1}^{m} e^{-c_{n} g_{j}}=(n / 2 \pi) \int_{0}^{\pi} e^{-c_{n} g(\omega)} d \omega+o(1) .
$$

Finally, write the first term on the right-hand side of (65) as $I_{1 n}+I_{2 n}$, where

$$
\begin{aligned}
& 2 \pi I_{1 n}=n \int_{\left|\omega-\omega_{0}\right| \geq \delta} e^{-c_{n} g(\omega)} d \omega, \\
& 2 \pi I_{2 n}=n \int_{\left|\omega-\omega_{0}\right|<\delta} e^{-c_{n} g(\omega)} d \omega .
\end{aligned}
$$

By assumption, we have

$$
g(\omega)-g\left(\omega_{0}\right) \geqq \eta \quad \text { for } \quad\left|\omega-\omega_{0}\right| \geqq \delta,
$$

where $\eta$ (depending on $\delta$ ) is positive. Hence

$$
2 \pi I_{1 n} \leqq n \pi e^{-\varepsilon_{n}\left\{\theta\left(\omega_{0}\right)+\eta\right\}}=O\left\{n(m / \sqrt{\log m})^{-\left(1+\eta / g\left(\omega_{0}\right)\right)}\right\}=O(1) .
$$

Also

$$
2 \pi I_{2 n}=n e^{-c_{n} g\left(\omega_{0}\right)} \int_{\left|\omega-\omega_{0}\right|<\delta} e^{-\frac{1}{2} c_{n}\left(\omega-\omega_{0}\right)^{2} g^{\prime \prime}\left(\omega_{1}\right)} d \omega,
$$

where $\left|\omega_{1}-\omega_{0}\right|<\delta$, by Taylor's theorem, and, using the fact that the integrand in (66) lies between $e^{-\frac{1}{2} c_{n} m_{0}}$ and $e^{-\frac{1}{2} c_{n} M_{0}}$ where $m_{0}, M_{0}$ are respectively the lower and upper bounds of $g^{\prime \prime}(\omega)$ in the region $\left|\omega-\omega_{0}\right|<\delta$, we find by a straightforward calculation that

and

$$
\varlimsup_{n \rightarrow \infty} I_{2 n} \leqq-\left(g^{\prime \prime}\left(\omega_{0}\right) / m_{0}\right)^{\frac{1}{2}} \log (1-P),
$$

$$
\varliminf_{n \rightarrow \infty} I_{2 n} \geqq-\left(g^{\prime \prime}\left(\omega_{0}\right) / M_{0}\right)^{\frac{1}{2}} \log (1-P) .
$$


But $M_{0}-m_{0}$ can be made arbitrarily small by taking $\delta$ to be sufficiently small, and so we must have

or, from (65),

$$
\lim _{n \rightarrow \infty}\left(I_{1 n}+I_{2 n}\right)=-\log (1-P),
$$

$$
\lim _{n \rightarrow \infty} \sum_{j=1}^{m} e^{-c_{n} \theta_{j}}=-\log (1-P)
$$

Hence, from (63),

$$
\lim _{n \rightarrow \infty} p\left\{\frac{1}{2} \max _{1 \leqq j \leqq m}\left(2 \pi f\left(\omega_{j}\right) I_{n, \varepsilon}\left(\omega_{j}\right)\right)>c_{n}\right\}=P,
$$

and the result follows immediately from the conclusion of Theorem 2 . If $\omega_{0}=0$ or $\pi$, the factor 2 multiplying $g\left(\omega_{0}\right)$ in the third term on the righthand side of (61) must be omitted.

Theorem 8 has corollaries analogous to those of Theorem 6; in the analogue of the second corollary it is easy to see that $c_{n}$ must be replaced by $c_{n} / \operatorname{var} x=c_{n} / \int_{-\pi}^{\pi} f(\omega) d \omega$, and that the simpler expression $2 \sum_{t=1}^{n} x_{t}^{2} / n$ can be used instead of $m^{-1} \sum_{j=1}^{m} I_{n, x}\left(\omega_{j}\right)$ or $([n / 2])^{-1} \sum_{j=0}^{[n / 2]} I_{n, x}\left(\omega_{j}\right)$. The result will also hold when in $(61) g\left(\omega_{0}\right), g^{\prime \prime}\left(\omega_{0}\right)$ are replaced by suitable parametric estimates $\hat{g}\left(\hat{\omega}_{0}\right), \hat{g}^{\prime \prime}\left(\hat{\omega}_{0}\right)$.

When $\varepsilon_{t}$ is not normal it should be possible to establish rigorously the analogue of Theorem 7 , according to which $p\left\{\frac{1}{2} \max _{1 \leqq j \leqq m} I_{n, x}\left(\omega_{j}\right)>c_{n}^{\prime}\right\}$, where $c_{n}^{\prime}$ is obtained by replacing $-\log (1-P)$ by $P$ in the formula $(61)$ for $c_{n}$, should lie between $P-\frac{1}{2} P^{2}$ and $P$ for sufficiently large $n$. This follows from the fact that

$$
2 \sum_{j>k=1}^{m} e^{-\sigma_{n}^{\prime}\left(g_{j}+\theta_{k}\right)}=\left(\sum_{j=1}^{m} e^{-c_{n}^{\prime} g_{j}}\right)^{2}-\sum_{j=1}^{m} e^{-2 c_{n}^{\prime} g_{s},}
$$

which can easily be shown to tend to $P^{2}$ when $n \rightarrow \infty$.

\section{The asymptotic distribution of the largest periodogram value for any angular frequency}

The corresponding problems when the argument of the periodogram function ranges over the whole interval $[0, \pi]$, namely the investigation of the asymptotic behaviour of

and

$$
I_{n, x}^{(m)}=\max _{0 \leqq \omega \leqq \pi} I_{n, x}(\omega)
$$

$$
K_{n, x}^{(m)}=\max _{0 \leqq \omega \leqq \pi} K_{n, x}(\omega),
$$

are of considerable interest; in fact for the two types of hypothesis testing 
problem referred to in $\S 4$ greater power would in general be achieved by using the statistics $I_{n, x}^{(m)}$ and $K_{n, x}^{(m)}$ respectively, and these statistics are what a method essentially the same as the maximum-likelihood ratio method produces in the case of normally distributed $\varepsilon_{i}$. However, both these problems seem to be extraordinarily difficult.

When the sequence $\left\{\alpha_{u}\right\}$ obeys the conditions of Theorems 3 or $3 \mathrm{a}$, so that $p \lim _{n \rightarrow \infty} \max _{0 \leqq \omega \leqq \pi}\left|I_{n, x}(\omega)-2 \pi f(\omega) I_{n, \varepsilon}(\omega)\right|=0$, and $f(\omega)$ is never zero, the asymptotic distributions of $I_{n, x}^{(m)}$ and $K_{n, x}^{(m)}$ ought to be the same as those of $\max _{0 \leqq \omega \leqq \pi}\left\{2 \pi f(\omega) I_{n, \varepsilon}(\omega)\right\}$ and $I_{n, \varepsilon}^{(m)}=\max _{0 \leqq \omega \leqq \pi} I_{n, \varepsilon}(\omega)$ respectively, but the essential difficulty lies in the determination of the latter. The best results that have so far been obtained are those of Whittle (1959), namely

$$
p \lim _{n \rightarrow \infty}\left\{I_{n, \varepsilon}^{(m)} / 2 \log n\right\}=1
$$

(which we have already mentioned), and

$$
p \lim _{n \rightarrow \infty}\left\{\max _{n \leqq \omega \leqq \pi}\left[2 \pi f(\omega) I_{n, \varepsilon}(\omega)\right] / 4 \pi f\left(\omega_{0}\right) \log n\right\}=1,
$$

when $f$ has a unique maximum at $\omega=\omega_{0}$.

Incidentally, (68) follows at once from (43) and Theorem 8 or the corresponding result for non-normal $\varepsilon_{t}$ by using the obvious inequalities

$$
\max _{1 \leqq j \leqq[(n-1) / 2]}\left\{2 \pi f(2 \pi j / n) I_{n, \varepsilon}(2 \pi j / n)\right\} \leqq \max _{0 \leqq \omega \leqq \pi}\left\{2 \pi f(\omega) I_{n, \varepsilon}(\omega)\right\} \leqq 2 \pi f\left(\omega_{0}\right) I_{n_{\varepsilon} \varepsilon}^{(m)}
$$

From (43), (68), and Theorem 3 or $3 a$, we obtain

$$
p \lim _{n \rightarrow \infty}\left\{I_{n, \varepsilon}^{(m)} / 4 \pi f\left(\omega_{0}\right) \log n\right\}=1,
$$

and

$$
p \lim _{n \rightarrow \infty}\left\{K_{n, x}^{(m)} / 2 \log n\right\}=1
$$

However, (69) and (70) are not very informative. What one really wants is some means of deriving approximations to values of $I_{n, x}^{(m)}$ or $K_{n, x}^{(m)}$ which are exceeded with some specified probability $P$, particularly when $P$ is small. It seems reasonable to conjecture that the only hope of discovering this lies in finding a way of strengthening the methods used by Whittle. The result (33) of Lemma 1 is of no use because of the factor $\log n$, which makes the conclusions that can be drawn from it much weaker than (69) or $(70)$. 


\section{Appendix}

\section{Effect of applying a correction for the series mean}

It is easily seen that all the results we have obtained will still hold with very minor modifications when $x_{t}$ is replaced throughout by $x_{t}-\bar{x}$, where $\bar{x}=\sum_{t=1}^{n} x_{t} / n$, so that instead of the model (2) we can have

$$
x_{t}=\mu+\sum_{u=0}^{\infty} \alpha_{u} \varepsilon_{t-u},
$$

where $\mu=E\left(x_{t}\right)$ is an unknown parameter.

For

$$
(2 / n)^{\frac{1}{2}} \sum_{t=1}^{n}\left(x_{t}-\bar{x}\right) e^{i \omega t}-J_{n, x}(\omega)=(2 / n)^{\frac{1}{2}} \bar{x} \sum_{t=1}^{n} e^{i \omega t}
$$

and since $\lim _{n \rightarrow \infty} n \operatorname{var} \bar{x}=2 \pi f(0)$ (see, for example, Hannan, 1960, p. 127),

$$
\left|\sum_{t=1}^{n} e^{i \omega t}\right|=\left|\sin \frac{1}{2} n \omega / \sin \frac{1}{2} \omega\right| \leqq \min \left(1, \frac{1}{2} n \omega\right) / \sin \frac{1}{2} \omega \quad(\omega \neq 0),
$$

(A1) is identically zero for $\omega=2 \pi j / n(j \neq 0)$, is $O_{p}\left(n^{\beta-1}\right)$ uniformly in $\omega$ for $n^{-\beta}<\omega \leqq \pi(\beta<1)$, and $O_{p}(1)$ uniformly in $\omega$ for $0 \leqq \omega \leqq \pi$. It follows that in Theorems $1,2,2 a, 2 b, 4,5,6,7$ and 8 , we need only exclude $\omega=0$ from the range of angular frequencies, while Theorems $3,3 \mathrm{a}, 3 \mathrm{~b}$ will remain true if the maximum is taken over the range defined by $n^{-\beta}<\omega \leqq \pi(\beta<1)$. Also (69) and (70) will still hold, but we should expect the asymptotic distribution of $K_{n, x}^{(m)}$ to be affected, although not that of $I_{n, x}^{(m)}$ unless the maximum of the spectral density $f$ occurs at $\omega_{0}=0$.

\section{References}

[1] Bartlett, M. S., An Introduction to Stochastic Processes, Cambridge University Press (1955).

[2] Cramér, H., Mathematical Methods of Statistics, Princeton (1946).

[3] Cramér, H., Random Variables and Probability Distributions, 2nd edn., Cambridge University Press (1962).

[4] Feller, W., An Introduction to Probability Theory and its Applications, Vol. I, 2nd edn., Wiley, New York (1957).

[5] Hannan, E. J., Time Series Analysis, Methuen (1960).

[6] Hannan, E. J., Testing for a jump in the spectral function, J. Roy. Statist. Soc. B, 23 (1961), 394-404.

[7] Priestley, M. B., Analysis of stationary processes with mixed spectra, J. Roy. Statist. Soc. B, $24(1962), 511-29$.

[8] Walker, A. M., Some tests of separate families of hypotheses in time series analysis. Unpublished paper (1964).

[9] Whittle, P., The simultaneous estimation of a time-series' harmonic components and covariance structure, Trab. estad. 3 (1952), 43-57. 
[10] Whittle, P., A Study in the Analysis of Stationary Time Series, Appendix 2. Uppsala: Almqvist and Wiksell (1954).

[11] Whittle, P., Sur la distribution du maximum d'un polynome trigonométrique à coefficients aléatoires, Colloques Internationaux du Centre National de la Recherche Scientifique, LXXXVII, Paris (1959), 173-84.

[12] Zygmund, A., Trigonometric Series. Vol. 2., Cambridge University Press (1959).

\section{University of Cambridge}

and

Institute of Advanced Studies, Australian National University. 